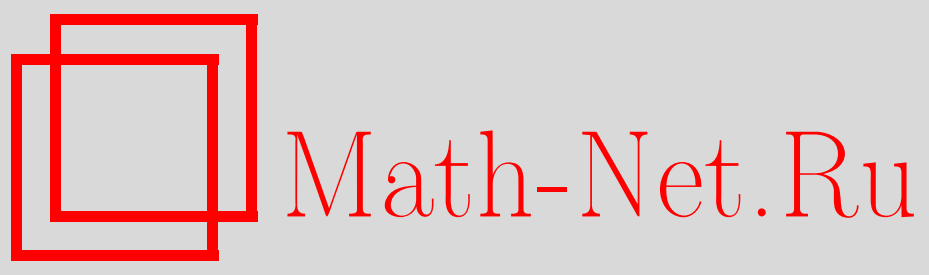

Д. В. Исангулова, Квазиконформные отображения группы движений плоскости, Матем. заметки, 2013, том 93, выпуск 6, 947-950

DOI: https://doi.org/10.4213/mzm10247

Использование Общероссийского математического портала Math-Net.Ru подразумевает, что вы прочитали и согласны с пользовательским соглашением http://www . mathnet.ru/rus/agreement

Параметры загрузки:

IP : 3.81 .55 .215

26 апреля 2023 г., $17: 48: 10$

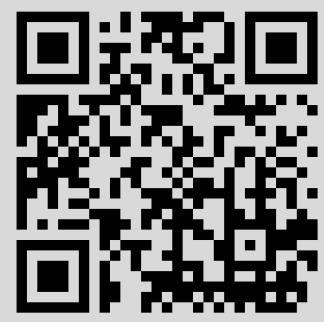




\section{Квазиконформные отображения группы движений плоскости}

\section{Д. В. Исангулова}

1. Введение. Мы исследуем квазиконформные отображения группы $S E(2)$ - группы движений евклидовой плоскости, сохраняющих ориентацию. Всякое сохраняющее ориентацию движение плоскости есть композиция сдвига на вектор $(x, y)$ и поворот на угол $\theta$ против часовой стрелки, поэтому $S E(2)$ еще называется группой поворотов-сдвигов. Это трехмерное топологическое многообразие, диффеоморфное $\mathbb{R}^{2} \times S^{1}$, с умножением

$$
\left(x_{0}, y_{0}, \theta_{0}\right) \cdot(x, y, \theta)=\left(x_{0}+x \cos \theta_{0}-y \sin \theta_{0}, y_{0}+x \sin \theta_{0}+y \cos \theta_{0}, \theta_{0}+\theta\right)
$$

и левоинвариантными векторными полями

$$
A=\cos \theta \frac{\partial}{\partial x}+\sin \theta \frac{\partial}{\partial y}, \quad B=\frac{\partial}{\partial \theta}, \quad C=-\sin \theta \frac{\partial}{\partial x}+\cos \theta \frac{\partial}{\partial y} .
$$

На $S E(2)$ мы рассматриваем субриманову структуру, задаваемую горизонтальным подрасслоением $H=\operatorname{span}\{A, B\}$ в касательном расслоении. Субриманова метрика или метрика Карно-Каратеодори $d$ задается как инфимум длин всех горизонтальных кривых, соединяющих две точки (кусочно-гладкая кривая называется горизонтальной, если ее касательный вектор принадлежит $H$ почти всюду). Для измерения длин горизонтальных кривых введем такое внутреннее произведение на $H$, при котором векторные поля $A$ и $B$ ортонормированы. По теореме Рашевского-Чоу метрика $d$ корректно определена, поскольку векторные поля $A$ и $B$ удовлетворяют условию Хёрмандера: $\operatorname{span}\{A, B,[A, B]=-C\}$ совпадает со всем касательным расслоением [1].

Группа движений плоскости с субримановой геометрией возникает в вопросах моделирования неголономного движения и оптимального контроля. Также геометрия группы $S E(2)$ возникает в задаче визуализации при моделировании восприятия человеческим мозгом плоского черно-белого изображения (см., например, монографию [2]). Субримановы геодезические вычислены в статье [3]. В работе автора [4] описаны $C^{2}$-гладкие изометрии на $S E(2)$.

Напомним метрическое определение квазиконформности: гомеоморфизм $F: \Omega \rightarrow \Omega^{\prime}$ связных областей $\Omega, \Omega^{\prime} \subset S E(2)$ называется $K$-квазиконформным, если

$$
\varlimsup_{r \rightarrow+0} \frac{\max _{\eta \in \Omega}\{d(F(\xi), F(\eta)) \mid d(\xi, \eta)=r\}}{\min _{\eta \in \Omega}\{d(F(\xi), F(\eta)) \mid d(\xi, \eta)=r\}} \leqslant K<\infty \quad \text { для всякого } \quad \xi \in \Omega .
$$

Первый вопрос, возникающий при исследовании нового класса отображений: насколько богат этот класс? Теорема 1 утверждает, что множество квазиконформных отображений на группе $S E(2)$ бесконечномерно.

Теорема 1. Пусть $q$-гладкая вещественнозначная функиия и

$$
k=\sup \sqrt{(A B q+B A q)^{2}+\left(A^{2} q-B^{2} q-q\right)^{2}}<+\infty .
$$

Тогда векторное поле $v=-B q A+A q B+q C$ порождает локальную однопараметрическую группу $K_{s}$-квазиконформных преобразований $F_{s}$, где

$$
\frac{1}{2}\left(K_{s}+\frac{1}{K_{s}}\right) \leqslant e^{\sqrt{2} k|s|} .
$$

Работа выполнена при поддержке программы "Ведущие научные школы" (грант № НШ921.2012.1) и ФЦП "Научные и научно-педагогические кадры инновационной России" на 20092013 гг. (соглашение № 8206).

DOI: $10.4213 / \mathrm{mzm} 10247$ 
ПримеР. Если $q=q(\theta)$ и $k=\sup \left|B^{2} q+q\right|<\infty$, то $F_{s}(x, y, \theta)=\left(f_{s}, g_{s}, \theta\right)$ с

$$
f_{s}=x-s(B q \cos \theta+q \sin \theta), \quad g_{s}=y-s(B q \sin \theta-q \cos \theta) .
$$

Причем

$$
\frac{1}{2}\left(K+\frac{1}{K}\right) \leqslant 1+\frac{s^{2} k^{2}}{2} \leqslant e^{\sqrt{2} k|s|} .
$$

Теорема 2 дает описание всех $C^{4}$-гладких 1-квазиконформных отображений на $S E(2)$.

Tеорема 2. Всякое $C^{4}$-гладкое 1-квазиконформное отображение на группе SE(2) изометрично и является композичией следующих отображений:

$$
\begin{array}{cc}
l_{\left(x_{0}, y_{0}, \theta_{0}\right)}(x, y, \theta)=\left(x_{0}, y_{0}, \theta_{0}\right) \cdot(x, y, \theta) & (\text { левъй сдвиг), } \\
\iota(x, y, \theta)=(-x, y,-\theta), \quad \sigma(x, y, \theta)=(-x,-y, \theta) & (\text { отражения). }
\end{array}
$$

Приведем кратко схемы доказательств теорем 1 и 2.

2. Доказательство теоремы 1. При доказательстве теоремы 1 мы опираемся на работу [5], где доказаны сходные факты для контактных и квазиконформных потоков группы Гейзенберга $\mathbb{H}^{1}$.

На группе $S E(2)$ определена контактная форма $\omega=-\sin \theta d x+\cos \theta d y$, согласованная с субримановой структурой: $\operatorname{ker} \omega=H$ и $\left(l_{\xi_{0}}\right)_{*} \omega=\omega$, где $l_{\xi_{0}}(\xi)=\xi_{0} \cdot \xi-$ левый сдвиг, $\xi_{0}, \xi \in S E(2)$. Как обычно, отображение $F=(f, g, \varphi)$ контактно, если $F^{*} \omega=\lambda \omega$. Отсюда получаем, что

$$
A g \cos \varphi=A f \sin \varphi, \quad B g \cos \varphi=B f \sin \varphi \quad \text { (условия контактности) }
$$

и $\lambda=\operatorname{det} D_{\mathrm{h}} F$, где $D_{\mathrm{h}} F(\xi): H_{\xi} \rightarrow H_{F(\xi)}$ - горизонтальный дифференциал, определяемый в базисе $\{A, B\}$ матрицей

$$
\left(\begin{array}{cc}
\frac{A f}{\cos \varphi} & \frac{B f}{\cos \varphi} \\
A \varphi & B \varphi
\end{array}\right) .
$$

При этом, как и в евклидовом случае, $D_{\mathrm{h}} l_{\xi_{0}} \equiv I$ и верно обратное: если $D_{\mathrm{h}} F \equiv I$, то $F$ - левый сдвиг.

Известно [6], что $K$-квазиконформное отображение $F$ hс-дифференцируемо почти всюду, контактно и

$$
\left\|D_{\mathrm{h}} F(\xi)\right\|_{1}^{2} \leqslant K\left|\operatorname{det}\left(D_{\mathrm{h}} F(\xi)\right)\right| \text { почти всюду, }
$$

где $\|\cdot\|_{1}$ - операторная норма матрицы.

Векторное поле $v$ генерирует поток отображений $F_{s}$, если $d F_{s} / d s=v \circ F_{s}$ и $F_{0}=$ id. Поле $v$ называется инфинитезималъным генератором потока $F_{s}$, причем $F_{s}$ будет (локальной) однопараметрической группой преобразований.

Векторное поле вида $v=-B q A+A q B+q C$, где $q$ - произвольная гладкая вещественнозначная функция, генерирует локальную однопараметрическую группу контактных преобразований; верно и обратное, всякое гладкое векторное поле $v$, которое генерирует локальную однопараметрическую группу контактных преобразований, имеет такой вид с $q=\langle\omega, v\rangle\left[4 ;\right.$ теорема 2]. Отметим, что $\lambda_{s}=\operatorname{det} D_{\mathrm{h}} F_{s}$ будет строго положительной.

Обозначим через $M_{s}$ матрицу $D_{\mathrm{h}} F_{s}$, а через $\alpha>\beta>0$ - сингулярные числа $M_{s}$. Тогда

$$
\left\|M_{s}\right\|_{2}^{2} \stackrel{\text { def }}{=} \operatorname{tr}\left(M_{s}^{t} M_{s}\right)=\alpha^{2}+\beta^{2}, \quad \operatorname{det} M_{s}=\alpha \beta, \quad \frac{\alpha}{\beta} \leqslant K .
$$

Имеем [4; лемма]

$$
\frac{d}{d s} M_{s}=V M_{s}, \quad \text { где } \quad V=\left(\begin{array}{cc}
-A B q & -B^{2} q-q \\
A^{2} q & B A q
\end{array}\right) .
$$


Тогда

где

$$
\frac{d}{d s} \frac{M_{s}^{t} M_{s}}{\operatorname{det} M_{s}}=\frac{1}{\operatorname{det} M_{s}}\left(M_{s}^{t}\left(V^{t}+V-\operatorname{tr} V \cdot I\right) M_{s}\right)=\frac{2}{\operatorname{det} M_{s}} M_{s}^{t} S M_{s},
$$

$$
S=\frac{1}{2}\left(\begin{array}{cc}
-A B q-B A q & A^{2} q-B^{2} q-q \\
A^{2} q-B^{2} q-q & A B q+B A q
\end{array}\right),
$$

и получаем оценку

$$
\frac{d}{d s} \frac{\left\|M_{s}\right\|_{2}^{2}}{\operatorname{det} M_{s}}=\frac{2}{\operatorname{det} M_{s}} \operatorname{tr}\left(M_{s}^{t} S M_{s}\right) \leqslant 2 \frac{\left\|M_{s}\right\|_{2}^{2}}{\operatorname{det} M_{s}}\|S\|_{2} .
$$

Окончательно получаем

$$
\frac{1}{2}\left(\frac{\alpha}{\beta}+\frac{\beta}{\alpha}\right) \leqslant e^{2 s\|S\|_{2}} \leqslant e^{\sqrt{2} k s}
$$

поскольку

$$
\|S\|_{2}=\frac{1}{2} \sqrt{2(A B q+B A q)^{2}+2\left(A^{2} q-B^{2} q-q\right)^{2}} \leqslant \frac{k}{\sqrt{2}} .
$$

3. Схема доказательства теоремы 2. Вначале мы находим 1-квазиконформные потоки. Для этого решаем систему дифференциальных уравнений

$$
A A q-B B q-q=0, \quad A B q+B A q=0 .
$$

Раскладывая функцию $q$ в ряд Фурье по переменной $\theta$ и предполагая, что $q \in C^{4}$, получаем, что

$$
q=\left(C_{1}+C_{0} x\right) \cos \theta+\left(C_{2}+C_{0} y\right) \sin \theta .
$$

Последнее соответствует левому сдвигу $F_{s}=l_{\left(a(s), b(s), s C_{0}\right)}$, где

$$
a(s)=\frac{-C_{1}+C_{1} \cos \left(s C_{0}\right)-C_{2} \sin \left(s C_{0}\right)}{C_{0}}, \quad b(s)=\frac{-C_{2}+C_{2} \cos \left(s C_{0}\right)+C_{1} \sin \left(s C_{0}\right)}{C_{0}},
$$

если $C_{0} \neq 0$, и

$$
a(s)=-s C_{2}, \quad b(s)=s C_{1},
$$

если $C_{0}=0$.

Далее, показываем, что всякое $C^{4}$-гладкое 1-квазиконформное отображение $F$ можно встроить в поток. Применяя левые сдвиги, можно считать, что существуют две окрестности единицы группы $U$ и $V$ такие, что $F(U)=V$.

Обозначим через $G_{s}$ гладкий поток 1-квазиконформных отображений. По вышеописанному $G_{s}$ есть поток левых сдвигов и $D_{\mathrm{h}} G_{s} \equiv I$.

Введем еще одно 1-квазиконформное отображение $\Phi_{s}=F^{-1} \circ G_{s} \circ F$. Так как $G_{0}=\mathrm{id}$ и оба отображения $F$ и $F^{-1}$ корректно определены в окрестности единицы группы, то при малых $s$ семейство $\Phi_{s}$ будет $C^{4}$-гладким потоком 1-квазиконформных отображений на некоторой окрестности единицы группы и, следовательно, $\Phi_{s}-$ поток левых сдвигов и $D_{\mathrm{h}} \Phi_{s} \equiv I$. Имеем

$$
D_{\mathrm{h}} G_{s}(F(\xi)) \cdot D_{\mathrm{h}} F(\xi)=D_{\mathrm{h}} F\left(\Phi_{s}(\xi)\right) \cdot D_{\mathrm{h}} \Phi_{s}(\xi) \quad \Longrightarrow \quad D_{\mathrm{h}} F(\xi)=D_{\mathrm{h}} F\left(\Phi_{s}(\xi)\right) .
$$

Фиксируем точку $\xi$ из окрестности единицы. При варьировании $G_{s}$ и $s$, множество точек $\Phi_{s}(\xi)$ совпадет с целой окрестностью единицы. Поэтому $D_{\mathrm{h}} F \equiv$ const. Нетрудно показать, что

$$
D_{\mathrm{h}} F \equiv \pm I \quad \text { или } \quad D_{\mathrm{h}} F \equiv\left(\begin{array}{cc}
-1 & 0 \\
0 & 1
\end{array}\right) .
$$

Во всех трех случаях $F$ изометрично и является суперпозицией отображений из теоремы 2. 


\section{СПИСОК ЦИТИРОВАННОЙ ЛИТЕРАТУРЫ}

[1] П. К. Рашевский, Уч. зап. Моск. гос. пед. ин-та им. К. Либкнехта. Сер. физ.-матем., 2 (1938), 83-94. [2] L. Capogna, D. Danielli, S. D. Pauls, J. T. Tyson, An Introduction to the Heisenberg Group and the Sub-Riemannian Isoperimetric Problem, Progr. Math., 259, Birkhäuser-Verlag, Basel, 2007. [3] I. Moiseev, Yu. L. Sachkov, ESAIM Control Optim. Calc. Var., 16:2 (2010), 380-399. [4] Д. В. Исангулова, Вестн. Кемеровского гос. ун-та, 2011, № 3/1 (47), 243-249. [5] A. Korányi, H. M. Reimann, Invent. math., 80:2 (1985), 309-338. [6] S. K. Vodopyanov, The Interaction of Analysis and Geometry, Contemp. Math., 424, Amer. Math. Soc., Providence, RI, 2007, 247-301.

\section{Д. В. Исангулова}

Поступило

Институт математики им. С. Л. Соболева СО РАН,

г. Новосибирск

E-mail: dasha@math.nsc.ru 